\title{
In Vitro Comparison of the Effect of Inhalation Delay and Flow Rate on the Emitted Dose from Three Valved Holding Chambers
}

\author{
Lois Slator, MSc, Dirk von Hollen, BS, ${ }^{1}$ Dennis Sandell, PhLic, ${ }^{3}$ and Ross H.M. Hatley, PhD ${ }^{1}$
}

\begin{abstract}
Background: Valved holding chambers (VHCs) are accessory devices for pressurized metered dose inhalers (pMDIs). Use of a VHC may help overcome coordination issues associated with drug delivery via the pMDI alone. Previous work has established that aspects of VHC use, including the time between actuation and inhalation (inhalation delay) and inhalation flow rate, can influence the amount of drug available to inhalation. This study compared the impact of inhalation delay and flow rate on the in vitro delivery of aerosol from different VHC brands.

Methods: A custom-built inhalation delay test rig, which enabled automation of controlled inhalation delays $(0,5$, or $10 \mathrm{sec}$ ), was developed. Extraction air flow was set to 5, 15, or $30 \mathrm{~L} / \mathrm{min}$. Delivery of albuterol (ProAir HFA $90 \mu \mathrm{g}$ ) to a filter (emitted dose) was assessed using three commercially available VHC brands (one conventional, two antistatic). Emitted dose under 27 different combinations of inhalation delay, flow rate, and VHC brand was determined in order to assess the effects of inhalation delay and flow rate. Pairwise comparisons of the different VHC brands with different inhalation delay/flow rate combinations were conducted to assess in vitro equivalence. Results: Emitted dose increased with flow rate and decreased with longer inhalation delays. Dependence on flow rate was similar for the two antistatic VHCs and more pronounced for the conventional VHC. The two antistatic VHCs showed equivalent results for the emitted dose of albuterol, across a range of flow rates and using different inhalation delays; the relation between the two antistatic VHCs fell within the $\pm 15 \%$ acceptance interval criteria for in vitro equivalence.

Conclusions: The different inhalation delays and flow rates had similar effects on the delivery of drug via the three VHCs. The two antistatic VHCs were shown to be equivalent in vitro in terms of emitted dose of albuterol.
\end{abstract}

Key words: valved holding chamber, inhalation delay, flow rate, in vitro equivalence, pMDI, emitted dose

\section{Introduction}

$\mathbf{V}$ ALVED HOLDING CHAMBERS (VHCs) are designed to overcome issues associated with drug delivery via pressurized metered dose inhalers (pMDIs). Use of a VHC can improve pulmonary targeting by reducing oropharyngeal impaction, and abrogate the detrimental effect of poor actuation-inhalation coordination on drug delivery. ${ }^{(1)}$ The presence of a valve allows the aerosol plume to be held temporarily within the $\mathrm{VHC},{ }^{(2)}$ which may negate the requirement for perfect actuation-inhalation coordination. In this sense, drug delivery via a VHC has been described as "partially breath-actuated."(3) Despite these advantages, use of a VHC cannot entirely overcome the need for coordination. Once actuated into the VHC, impaction, gravitational sedimentation, and electrostatic attraction act on the aerosol particles, which, over time, can reduce the amount of drug available to inhalation. ${ }^{(4)}$ Within-chamber impaction of nonrespirable particles is a putative mechanism by which VHCs improve the respirable profile of aerosol. ${ }^{(3)}$

In the presence of a long delay between pMDI actuation and inhalation, the gravitational sedimentation of aerosol particles may have a detrimental effect on aerosol delivery. Inhalation delays as short as $5 \mathrm{sec}$ have been shown to

\footnotetext{
${ }^{1}$ Respironics Respiratory Drug Delivery (UK) Ltd., a business of Philips Electronics UK Limited, Chichester, West Sussex, UK.

${ }^{2}$ Respironics, Inc., a Philips Healthcare company, Murrysville, PA, USA.

${ }^{3}$ S5 Consulting, Ekvägen 8, S-275 62 Blentarp, Sweden.
} 
significantly reduce respirable output, ${ }^{(5)}$ and inhalation delays of $30 \mathrm{sec}$ were shown to reduce patients' forced expiratory volume in $1 \mathrm{sec}$ response to terbutaline. ${ }^{(6)}$ However, the sedimentation rate of particles with a mass median aerodynamic diameter in the respirable range is likely to be very slow $(0.7 \mathrm{~mm} / \mathrm{sec}) .{ }^{(7)}$ This implies that after $30 \mathrm{sec}$, particles within $21 \mathrm{~mm}$ of the base of the VHC will have settled, such that sedimentation would have a greater effect on small-volume VHCs compared with large-volume VHCs.

Electrostatic charge can develop on the surface of VHCs, particularly on conventional devices, which are manufactured from nonconducting plastics. Electrostatic attraction between aerosol particles and the charged surface of the VHC retains aerosol within the device. ${ }^{(8)}$ Consequently, drug delivery via a conventional VHC has been shown to be inversely proportional to the static charge on the surface of the device. ${ }^{(9)}$ An inhalation delay increases the amount of time that aerosol must remain suspended within the chamber, where interactions with electrostatic charge can reduce aerosol delivery. ${ }^{(10)}$ VHCs that are manufactured from charge-dissipative polymers are less susceptible to the accumulation of electrostatic charge, and these antistatic VHCs have been shown to deliver a greater, more consistent dose of drug, with a higher respirable fraction. ${ }^{(11)}$ Given that antistatic VHCs have fewer forces acting to reduce aerosol delivery, the delivery from an antistatic VHC should also be less sensitive to the presence of an inhalation delay.

Inhalation technique, including inspiratory flow rate, can also influence drug delivery via a VHC. A slow $(30 \mathrm{~L} / \mathrm{min})$ inhalation followed by a $10-\mathrm{sec}$ breath-hold has been shown to improve lung deposition, ${ }^{(12)}$ and this technique is generally recommended. ${ }^{(13)}$ However, many patients, including infants and young children, inhale aerosol using tidal breaths and are unable to achieve a steady $30 \mathrm{~L} / \mathrm{min}$ flow rate. Despite this, these patients still benefit from inhaling aerosol via a VHC in several tidal breaths. ${ }^{(14,15)}$ Although previous research has established the effect of static charge and inhalation delay on aerosol delivery, little attention has been paid to the interaction between inhalation flow rates, static charge, and inhalation delays on aerosol delivery.

Previous in vitro work has characterized the performance of the OptiChamber Diamond VHC when used to deliver aerosol produced by two different pMDI brands at 15 and 30 $\mathrm{L} / \mathrm{min}$ extraction flow rates, without an inhalation delay. ${ }^{(16)}$ The present article reports on the interaction between clinically relevant inhalation delays $(0,5$, and $10 \mathrm{sec})$ and extraction flow rates $(5,15$, and $30 \mathrm{~L} / \mathrm{min})$, using a conventional VHC and two brands of antistatic VHC. The dose of albuterol delivered to a filter (emitted dose) was determined for each of the combinations of inhalation delay, flow rate, and VHC brand. The objectives of the study were: (i) to assess the effects of inhalation delay and flow rate, and to determine whether these effects differed between VHC brands; and (ii) to undertake pairwise comparisons between the three VHCs. These comparisons were intended to indicate whether the VHCs tested could be considered equivalent under the different in vitro test conditions.

\section{Materials and Methods}

\section{Test products}

Two brands of antistatic VHC, the OptiChamber Diamond VHC (Diamond; Respironics Respiratory Drug Delivery (UK) Ltd., a business of Philips Electronics UK Limited, Chichester, West Sussex, UK) and the AeroChamber Plus Z-Stat VHC (AC Z-Stat; Monaghan Medical Corp., Plattsburgh, NY), were compared with a conventional "static" brand of VHC, the AeroChamber Plus VHC (AC Plus; Monaghan Medical Corp.) (Fig. 1). Ten VHCs of each brand were tested. Forty-five suspension-based 200-dose albuterol (salbutamol) pMDIs (ProAir $90 \mu \mathrm{g}$ HFA; Teva Respiratory, LLC, Horsham, PA) were used to compare the three VHC brands (five pMDIs for each of the nine inhalation delay/flow rate combinations).

\section{Pretest conditions}

Prior to use, all equipment and fluids were stabilized to ambient conditions for $2 \mathrm{hr}$. A mixed anionic/nonionic detergent (15-30\% anionic surfactants, 5-15\% nonionic surfactants) was used to create the warm soapy water in which the components were washed (Fairy Liquid; Proctor \& Gamble UK, Weybridge, Surrey, UK).

Each VHC was washed with detergent, immersed in tap water, rinsed once under running fresh water, and left to air-dry in a drying cabinet. The pMDIs were primed, by actuating them three times to waste, before they were inserted into the experimental apparatus. Each pMDI actuator was washed, rinsed with deionized water, and air-dried, both before initial use and before each run during the study. Once dry, the canister was returned to the actuator, and the pMDI was primed using a single actuation. For each of the 45 pMDIs, the canister was used only with its supplied actuator.

\section{Test plan}

All tests were conducted according to a set test matrix, which was designed to take into account the possible effect of any systematic dose level trend through the life of the pMDI. Ten VHCs of each brand were assessed using five pMDIs, for each flow rate and delay time combination.

The same experimental design was used for all nine inhalation delay/flow rate combinations, and the same VHC units (10 of each type) were used in each of the nine investigations.
FIG. 1. Test components; pMDI and VHCs.

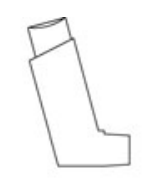

$45 \times$ ProAir HFA pMDI

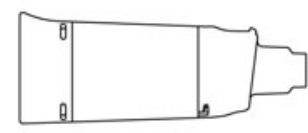

$10 \times$ OptiChamber

Diamond VHC

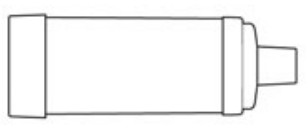

$10 \times$ AeroChamber Plus VHC

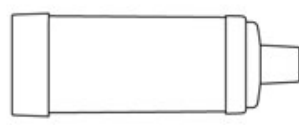

$10 \times$ AeroChamber Plus Z-Stat VHC 


\section{Custom-built inhalation delay test rig}

A custom-built inhalation delay test rig was developed to allow the automation and control of the inhalation delays (Fig. 2). Incorporation of a shutter gate allowed two configurations: "delay" and "inhalation." In delay configuration, the shutter gate was acted on by a compressed spring, but held closed by a solenoid. Activation of the solenoid facilitated rapid shutter gate opening (inhalation configuration). An extraction air flow was set at a rate of 5, 15, or $30 \mathrm{~L} / \mathrm{min}$. The VHCs were connected to the custom-built inhalation delay test rig using a custom adapter. The adapter was sealed with Parafilm M (Bemis Company, Inc., Oshkosh, WI). Air was extracted through the VHC to a low dead-space filter holder [Respironics Respiratory Drug Delivery (UK) Ltd.] and filter (67-mm 3M G-200 Filtrete ${ }^{\mathrm{TM}}$ electrostatic filter; 3M Filtration Products, St. Paul, MN). In the delay configuration, the extraction flow circulated via a built-in air bypass channel, which prevented air within the VHC being disturbed. The custom rig also featured a delay timer (H5CR Multifunction Digital Timer; Omron Corp., Osaka, Japan), which was programmed with the chosen inhalation delay. An ultrasonic microphone sensor (Knowles Acoustics, Itasca, IL) was attached to the pMDI with a Velcro band. The microphone detected actuation, triggering the start of the delay timer. Once the inhalation delay had elapsed, the shutter gate opened and air was drawn through the VHC to the collection filter.

Quantification of the delay test rig showed that the actual delay between actuation and the opening of the shutter gate was $0.034-0.094 \mathrm{sec}$. Flow rate was observed to take a further $0.123-0.255 \mathrm{sec}( \pm 10 \%)$ to establish. Total delay was therefore no more than the programmed delay, plus a maximum of 0.349 -sec intrinsic delay. Delay times presented are rounded to the nearest second.

\section{Experimental procedure}

Prior to each experiment, the delay timer was set to the relevant time interval and the extraction flow rate was preset. The test rig was activated, and the ultrasonic microphone sensor was attached to the actuator of a dummy pMDI. The sensitivity of the ultrasonic microphone sensor was gradually increased until it was set so that it was activated by actuation of the dummy pMDI. The filter was placed in a low dead-space filter holder and connected to the sliding shutter gate on the side with the air bypass channel (low dead-space filter holder with filter, Fig. 2). The other side of the filter holder was connected to a high-capacity vacuum pump (Copley HCP5; Copley Scientific Ltd., Nottingham, UK), and the exhaust of this pump was connected to the local exhaust ventilation extraction.

The TSI mass flow meter (TSI Incorporated, Shoreview, $\mathrm{MN}$ ) was switched on, reset to 0 , then connected between the VHC and test rig using a custom connector. The pMDI actuator mouthpiece was placed into the inlet of the VHC. The high-capacity vacuum pump was switched on and adjusted to achieve the predetermined flow rate. The TSI mass flow meter was then removed from the sliding gate, and the VHC was attached in its place via the mouthpiece adapter.

The ultrasonic sensor was fitted around the actuator of the pMDI in use, and the electronic delay timer was set to the required inhalation delay. The pMDI canister was removed from the actuator, shaken, and replaced. The sliding shutter gate was then closed (delay configuration, Fig. 2), and the pMDI actuated.

The sliding shutter gate opened automatically after the set inhalation delay (inhalation configuration, Fig. 2). Air was drawn through the VHC for $10 \mathrm{sec}$, after which the sliding shutter gate was manually closed. The pMDI canister was removed, shaken, and replaced.
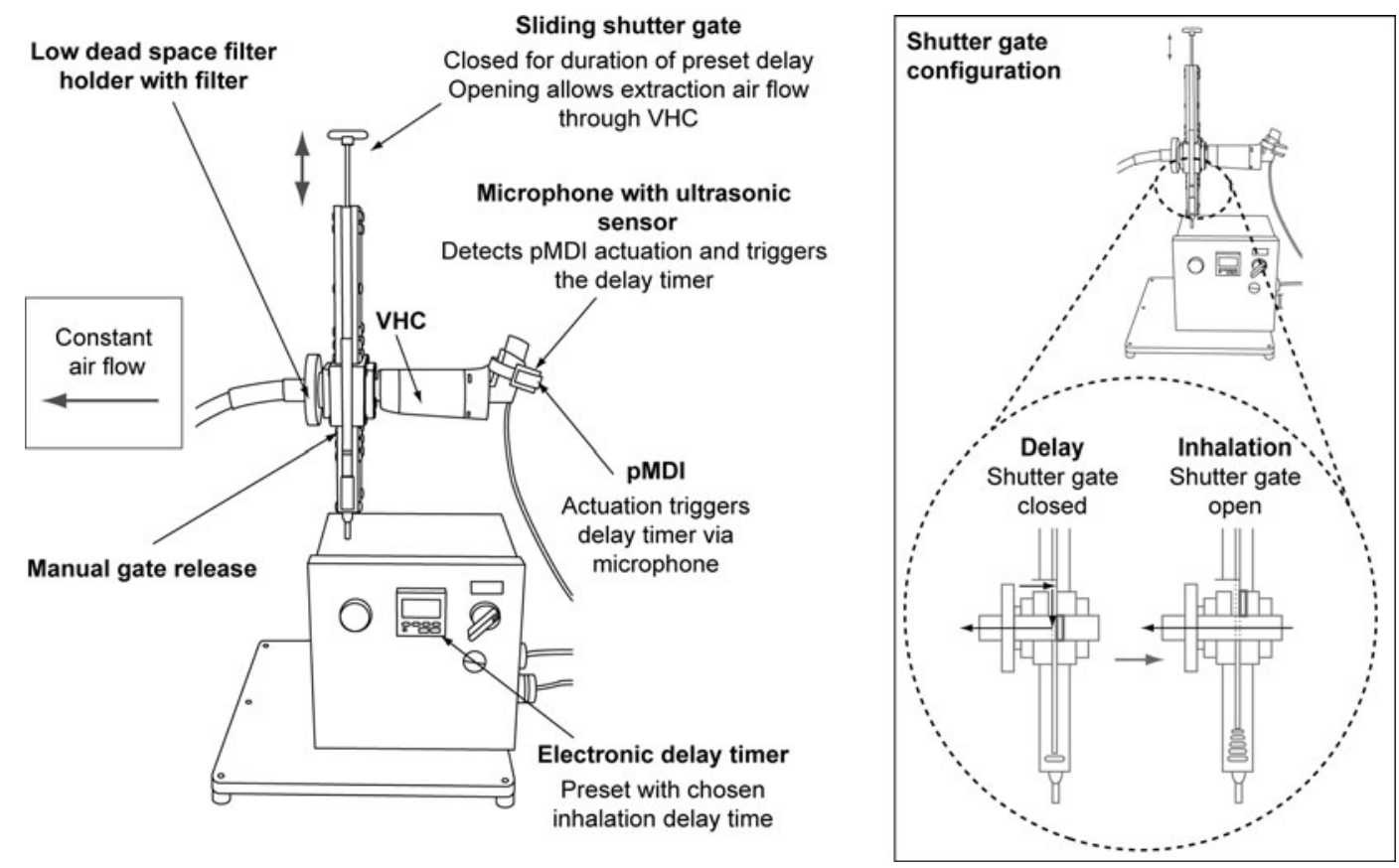

FIG. 2. Custom-built inhalation delay test rig. 
The procedure was repeated a further nine times, to give a total of 10 actuations per filter; this number of actuations was chosen to ensure that the lower limit of quantitation was reached. One filter was collected for each of the $10 \mathrm{VHCs}$ of each of the three brands. The amount of drug deposited within the VHC was determined after each sequence of 10 actuations.

Following each test, the filter was placed in a $50-\mathrm{mL}$ centrifuge tube. The filter holder was attached to an elastomeric-lipped ISO connector (Intersurgical Ltd., Wokingham, UK) and placed over the centrifuge tube. An appropriate diluent $(10 \%$ acetonitrile solution, $25 \mathrm{~mL})$ was dispensed onto the inside surface of the filter holder to wash off any residual drug. The diluent was collected in the centrifuge tube, and the rinsed filter holder was removed. The tube, with filter and diluent, was then centrifuged at $2,800 \mathrm{rpm}$ for $2 \mathrm{~min}$, inverted, then centrifuged again at $2,800 \mathrm{rpm}$ for $2 \mathrm{~min}$. The centrifuge process was repeated, so that each tube was centrifuged a total of four times.

The amount of drug in eluate extracted from the filter was quantified using high-performance liquid chromatography (HPLC; see HPLC analysis).

\section{HPLC analysis}

The HPLC system (Agilent 1100 series; Agilent Technologies, Waldbronn, Germany) was fitted with the appropriate column (Genesis Phenyl, $4 \mu \mathrm{m}, 150 \times 4.5 \mathrm{~mm}, 22^{\circ} \mathrm{C}$, $1 \mathrm{~mL} / \mathrm{min})$. Bracketing standards were made up and run, and the accuracy of the standards was checked. System suitability was then checked using five replicate injections of the bracketing standard to be used throughout the run. In order for the results of the run to be considered valid, the percentage relative standard deviation of bracketing standards had to be below $2 \%$ throughout the run, and the tailing factor (US Pharmacopeia method) had to be no greater than 2.0.

\section{Statistical analysis}

The dosing properties of the VHCs, under different delivery conditions, were compared using a regression model, in which flow rate $(5,15,30 \mathrm{~L} / \mathrm{min})$, inhalation delay $(0,5$, $10 \mathrm{sec})$, and VHC type were treated as fixed factors, and the pMDI $(1,2,3, \ldots 45)$ was treated as a random factor. Residuals were assumed to be approximately independent and normally distributed, with equal variance. The response variables were log transformed before the model fit; based on results of the model fit, estimated dose levels (geometric means), relative differences, and corresponding confidence intervals were calculated by back-transformation of the parameter estimates.

Results are reported in the form of geometric mean emitted dose, and emitted dose as a percentage of label claim. Estimated mean relative differences, which provide between-VHC comparisons, are reported for emitted dose (Table 2). Special attention was given to the $90 \%$ confidence interval $\left(\mathrm{CI}_{90}\right)$ for the estimated relative difference in emitted dose between the VHC brands. This is based on a regulatory requirement for a claim of in vitro equivalence between a "Test" and "Reference" product: if a $\mathrm{CI}_{90}$ for the mean Test/Reference ratio is within $\pm 15 \%$ (i.e., an acceptance interval of $85-118 \%$ ), in vitro equivalence can be concluded. ${ }^{(17)}$

\section{Results}

The mean emitted dose collected under different combinations of flow rate, inhalation delay, and VHC brand are compared in Table 1. Data relating to in vitro equivalence (confidence limits and $p$ values for between-VHC comparisons of emitted dose, under each flow rate/inhalation delay combination) are summarized in Table 2. Figures 3-5 illustrate the emitted dose, as a percentage of label claim, delivered under each of the flow rate/inhalation delay conditions, for each VHC brand. Mean emitted dose across the flow rates is also indicated.

There was a positive correlation between emitted dose and flow rate, such that higher flow rates were associated with greater emitted doses. This relationship was more pronounced with the AC Plus VHC, as indicated by the lower emitted dose achieved at $5 \mathrm{~L} / \mathrm{min}$ flow rate, compared with either the Diamond VHC or the AC Z-Stat VHC, which showed more similarity. The emitted dose was typically reduced when the inhalation delay was increased (Table 1).

\section{Between-VHC brand emitted dose comparisons; $5 \mathrm{~L} / \mathrm{min}$}

At $5 \mathrm{~L} / \mathrm{min}$ flow rate, with 0 -sec inhalation delay, the $\mathrm{CI}_{90}$ for all between-VHC brand comparisons fell outside the acceptance interval (85-118\%); all differences were statistically significant $(p<0.05)$, with the exception of the Diamond VHC versus AC Z-Stat VHC (Table 2). The corresponding $\mathrm{CI}_{90}(101-119 \%)$ for this comparison was marginally outside the acceptance interval. Overall, the Diamond VHC and the AC Z-Stat VHC delivered a higher emitted dose, compared with the AC Plus VHC. When a 5 -sec inhalation delay was incorporated, this pattern changed; a significantly lower dose was delivered via the $\mathrm{AC}$ Plus VHC, whereas the antistatic VHCs met the criteria for in vitro equivalence. The $\mathrm{CI}_{90}$ for the comparison of either antistatic VHC with the AC Plus VHC fell outside of the acceptance interval.

When the inhalation delay was increased to $10 \mathrm{sec}$, the Diamond VHC was again equivalent to the AC Z-Stat VHC. The emitted doses from the antistatic VHCs were significantly greater than those delivered via the conventional $\mathrm{AC}$ Plus VHC (Tables 1 and 2).

Table 1. Mean Emitted Dose (\% Label Claim) FOR EACH TEST CONDITION

\begin{tabular}{lcccc}
\hline & & \multicolumn{3}{c}{ Flow rate (L/min) } \\
\cline { 3 - 5 } VHC & Delay (sec) & 5 & 15 & 30 \\
\hline Diamond & 0 & 83.4 & 85.2 & 87.4 \\
Diamond & 5 & 69.5 & 76.4 & 80.6 \\
Diamond & 10 & 65.1 & 69.5 & 72.0 \\
AC Z-Stat & 0 & 76.1 & 91.7 & 90.1 \\
AC Z-Stat & 5 & 66.0 & 77.1 & 81.0 \\
AC Z-Stat & 10 & 65.0 & 72.8 & 75.5 \\
AC Plus & 0 & 61.4 & 79.4 & 85.8 \\
AC Plus & 5 & 50.9 & 62.5 & 77.1 \\
AC Plus & 10 & 46.0 & 62.8 & 61.9 \\
\hline
\end{tabular}


Table 2. Percent Estimated Relative Difference in Emitted Dose, 90\% Confidence Limits, and $P$ Values by Flow Rate, Inhalation Delay, and VHC Brand

\begin{tabular}{|c|c|c|c|c|c|c|c|}
\hline$V H C$ & Flow rate $(L / \mathrm{min})$ & & & & & & \\
\hline \multirow[t]{3}{*}{ Diamond } & 5 & $\begin{array}{c}136 \\
125-147 \\
(p<0.0001)\end{array}$ & $\begin{array}{c}136 \\
126-148 \\
(p<0.0001)\end{array}$ & $\begin{array}{c}141 \\
130-153 \\
(p<0.0001)\end{array}$ & $\begin{array}{c}110 \\
101-119 \\
(p=0.0627)\end{array}$ & $\begin{array}{c}105 \\
97-114 \\
(p=0.2972)\end{array}$ & $\begin{array}{c}100 \\
92-109 \\
(p=0.9684)\end{array}$ \\
\hline & 15 & $\begin{array}{c}107 \\
99-116 \\
(p=0.1504)\end{array}$ & $\begin{array}{c}122 \\
113-132 \\
(p<0.0001)\end{array}$ & $\begin{array}{c}111 \\
102-120 \\
(p=0.0367)\end{array}$ & $\begin{array}{c}93 \\
86-101 \\
(p=0.1371)\end{array}$ & $\begin{array}{c}99 \\
91-107 \\
(p=0.8494)\end{array}$ & $\begin{array}{c}96 \\
88-104 \\
(p=0.3518)\end{array}$ \\
\hline & 30 & $\begin{array}{c}102 \\
94-111 \\
(p=0.6910)\end{array}$ & $\begin{array}{c}105 \\
96-113 \\
(p=0.3669)\end{array}$ & $\begin{array}{c}116 \\
107-126 \\
(p=0.0022)\end{array}$ & $\begin{array}{c}97 \\
90-105 \\
(p=0.5452)\end{array}$ & $\begin{array}{c}99 \\
92-108 \\
(p=0.9138)\end{array}$ & $\begin{array}{c}95 \\
88-103 \\
(p=0.3410)\end{array}$ \\
\hline AC Z-Stat & 30 & $\begin{array}{c}124 \\
114-134 \\
(p<0.0001) \\
115 \\
107-125 \\
(p=0.0034) \\
105 \\
97-114 \\
(p=0.3145)\end{array}$ & $\begin{array}{c}130 \\
120-140 \\
(p<0.0001) \\
123 \\
114-134 \\
(p<0.0001) \\
105 \\
97-114 \\
(p=0.3105)\end{array}$ & $\begin{array}{c}141 \\
130-153 \\
(p<0.0001) \\
116 \\
107-126 \\
(p=0.0025) \\
122 \\
112-132 \\
(p<0.0001)\end{array}$ & & & \\
\hline $\begin{array}{l}\text { Delay }(\mathrm{sec}) \\
\text { VHC }\end{array}$ & & 0 & $\begin{array}{c}5 \\
\text { AC Plus }\end{array}$ & 10 & 0 & $\stackrel{5}{\text { AC }}$ Z-Stat & 10 \\
\hline
\end{tabular}

Shaded cells indicate comparisons for which the $90 \%$ confidence interval falls within the $85-118 \%$ bracket.

Between-VHC brand emitted dose comparisons; $15 \mathrm{~L} / \mathrm{min}$

At $15 \mathrm{~L} / \mathrm{min}$ flow rate, following a 0 -sec inhalation delay, the dose emitted via the Diamond VHC and AC Z-Stat VHC met the criteria for in vitro equivalence. Emitted dose was also equivalent for the Diamond VHC versus the AC Plus VHC (Table 2).

Following a 5-sec inhalation delay, emitted dose delivered using the AC Plus VHC was lower than that delivered via the two antistatic VHCs. The two antistatic VHCs met the criteria for in vitro equivalence. In contrast, there was a significant difference between the dose emitted from the Diamond VHC versus the AC Plus VHC, and the AC Z-Stat VHC versus the AC Plus VHC.

Following a 10-sec inhalation delay, the Diamond VHC versus AC Plus VHC, and AC Z-Stat VHC versus AC Plus

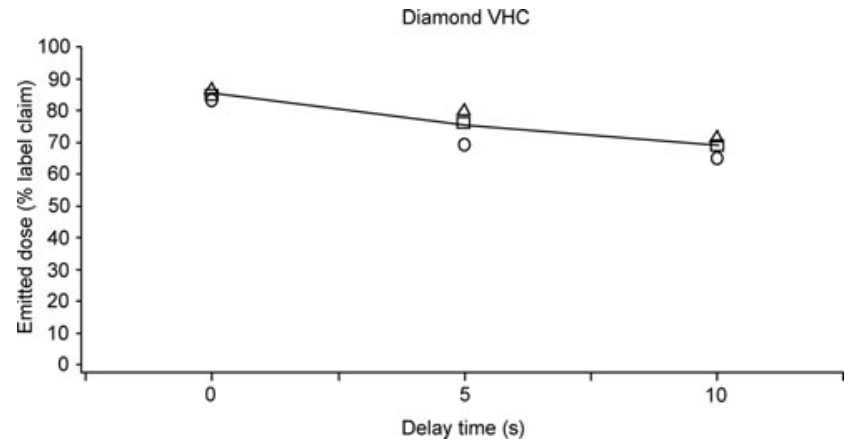

FIG. 3. Emitted dose, as a percentage of label claim, delivered via the Diamond VHC under each flow rate/inhalation delay combination: $\bigcirc$, emitted dose at $5 \mathrm{~L} / \mathrm{min} ; \square$, emitted dose at $10 \mathrm{~L} / \mathrm{min} ; \Delta$, emitted dose at $30 \mathrm{~L} / \mathrm{min} ;-$, mean emitted dose across flow rates.
VHC, showed a significant difference and had corresponding $\mathrm{CI}_{90}$ values that fell outside of the acceptance interval. The Diamond VHC versus AC Z-Stat VHC did not differ significantly, and therefore showed in vitro equivalence.

\section{Between-VHC brand emitted dose comparisons; $30 \mathrm{~L} / \mathrm{min}$}

When a $30 \mathrm{~L} / \mathrm{min}$ extraction flow rate with 0 -sec delay was used, none of the pairwise comparisons differed significantly. Given that all pairwise comparisons had $\mathrm{CI}_{90}$ values that fell within the acceptance interval, under these in vitro conditions, the three VHCs can be considered equivalent. Similarly, in the presence of a 5-sec inhalation delay, none of the pairwise comparisons had a $\mathrm{CI}_{90}$ that approached statistical significance.

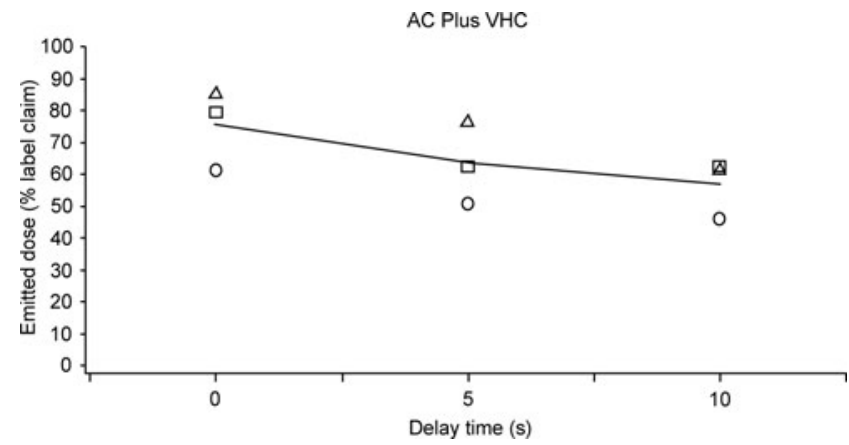

FIG. 4. Emitted dose, as a percentage of label claim, delivered via the AC Plus VHC under each flow rate/inhalation delay combination: $\bigcirc$, emitted dose at $5 \mathrm{~L} / \mathrm{min}$; $\square$, emitted dose at $10 \mathrm{~L} / \mathrm{min} ; \Delta$, emitted dose at $30 \mathrm{~L} / \mathrm{min} ;-$, mean emitted dose across flow rates. 


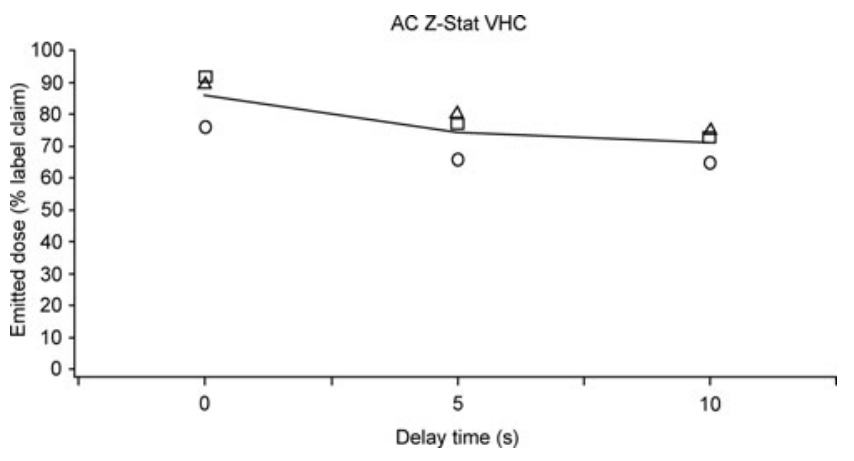

FIG. 5. Emitted dose, as a percentage of label claim, delivered via the AC Z-Stat VHC under each flow rate/inhalation delay combination: $\bigcirc$, emitted dose at $5 \mathrm{~L} / \mathrm{min}$; $\square$, emitted dose at $10 \mathrm{~L} / \mathrm{min} ; \Delta$, emitted dose at $30 \mathrm{~L} / \mathrm{min} ;-$, mean emitted dose across flow rates.

With a 10-sec inhalation delay, only the comparison of the Diamond VHC with the AC Z-Stat VHC demonstrated in vitro equivalence. The remaining comparisons showed statistically significant differences and had $\mathrm{CI}_{90}$ values that fell outside of the acceptance interval. Thus, of the VHCs tested, at $30 \mathrm{~L} / \mathrm{min}$ flow rate, only the Diamond VHC versus the AC Z-Stat VHC showed in vitro equivalence, regardless of the inhalation delay tested.

\section{Effect of inhalation delay on emitted dose}

Emitted dose correlated negatively with inhalation delay at each of the flow rates tested (Figs. 3-5). Between-VHC comparisons across the different inhalation delay/flow rate conditions indicated that the AC Z-Stat VHC and the Diamond VHC performed comparably. In addition, both the AC Z-Stat VHC and the Diamond VHC were relatively unaffected by changes to flow rate and inhalation delay, compared with the conventional AC Plus VHC. Variability in emitted dose was also lower with the AC Z-Stat VHC and the Diamond VHC, compared with the AC Plus VHC.

\section{Discussion}

For the purpose of this study, emitted dose was considered to be representative of the dose of albuterol available to inhalation. The variables included in this article were intended to represent drug delivery under a range of clinically relevant conditions. The results showed equivalence for the Diamond VHC with the predicate device, the AC Z-Stat VHC. ${ }^{(16)}$

Emitted dose was representative of the dose of drug available to inhalation via each VHC, when ProAir HFA was used under the conditions tested. Our results indicated that for all three VHCs, the emitted dose was reduced when the length of the inhalation delay increased. This supports the need for some degree of coordination, regardless of VHC use. For all VHCs, irrespective of extraction flow rate, the emitted dose after 10 -sec inhalation delay was approximately $80 \%$ of that delivered when no inhalation delay was incorporated.

The emitted dose increased with flow rate, and the relative increase was similar between inhalation delays, but different between VHCs. For both the Diamond VHC and the AC Z-Stat VHC, the emitted dose increased by 10-20\% when the extraction flow increased from 5 to $30 \mathrm{~L} / \mathrm{min}$; for the "static" AC Plus VHC, the corresponding increase was approximately $40 \%$.

Only the Diamond VHC and the AC Z-Stat VHC consistently fulfilled the $\pm 15 \%$ acceptance interval criteria for in vitro equivalence, across inhalation delays and flow rates, with a marginal exception for the $5 \mathrm{~L} / \mathrm{min}$ results with 0 -sec inhalation delay. Given that these were the only two antistatic VHCs in the experimental protocol, this was not surprising. At the higher flow rate of $30 \mathrm{~L} / \mathrm{min}$, and when inhalation delay was short $(5 \mathrm{sec})$ or absent $(0 \mathrm{sec})$, a greater number of pairwise VHC comparisons demonstrated performance equivalence in terms of emitted dose. This implies that the conventional AC Plus VHC can deliver a dose that is comparable to those of antistatic VHCs only under the more favorable conditions of shorter inhalation delays and higher flow rates.

Although the AC Plus VHC was washed prior to use in the study, it is plausible that the VHC material, which is more susceptible to the development of static charge, may confer a greater vulnerability to differences in conditions of use, such as flow rate and inhalation delay. It is wellestablished that, when a large number of doses are administered via a conventional VHC, the static charge on the VHC declines. ${ }^{(9)}$ This effect is known as VHC priming. In the present study, 10 consecutive actuations were performed. It is possible that this aspect of the study design would produce results that would minimize the issues associated with the use of conventional $\mathrm{VHCs}$, due to the mitigating effect of coating the internal surface of the VHC with aerosol.

The respirable proportion of the emitted dose, and the proportion that would impact in the oropharynx, were not determined in the present study. Previous work using radiolabeled aerosol established that "low" flow rates $(30 \mathrm{~L} /$ min) can achieve more favorable lung deposition, whereas higher flow rates $(90 \mathrm{~L} / \mathrm{min})$ were associated with greater degrees of oropharyngeal impaction. ${ }^{(12)}$ A low flow rate of $30 \mathrm{~L} / \mathrm{min}$ was found to be optimal, consistent with the work presented here. It is therefore unlikely that the emitted dose would consist of predominantly nonrespirable aerosol.

\section{Conclusions}

This article has reported the results of an in vitro investigation into the effect of inhalation delays, and clinically relevant flow rates, on the delivery of albuterol via different types (conventional or antistatic) and brands of VHC. The results indicated that the emitted dose increased with flow rate and decreased with longer inhalation delays. Flow rate dependence was similar for the two antistatic VHCs, the Diamond VHC and the AC Z-Stat VHC, and was less pronounced than that observed with the "static" VHC, the AC Plus VHC. For all VHCs tested, the emitted dose was reduced by approximately $20 \%$ as the inhalation delay was extended from 0 to $10 \mathrm{sec}$. In conclusion, the two antistatic VHCs, the Diamond VHC and the AC Z-Stat VHC, show equivalence in terms of the emitted dose of ProAir HFA, delivered across a range of flow rates, and using different inhalation delays; this equivalence fulfills the $\pm 15 \%$ acceptance interval criteria for in vitro equivalence.

\section{Acknowledgments}

The authors acknowledge Adam Metcalf of Respironics Respiratory Drug Delivery (UK) Ltd., a business of Philips 
Electronics UK Limited, Chichester, West Sussex, UK, for his analytical assistance, and Kurt Nikander, for his technical assistance and guidance. We also acknowledge PS5 Consultants Ltd., Portsmouth, UK, for drafting (Clare Nicholls) and editorial assistance (Stephen Keen). The study was sponsored by Respironics Respiratory Drug Delivery (UK) Ltd.

\section{Author Disclosure Statement}

Lois Slator, Dirk von Hollen, and Ross Hatley are all employees of Respironics Respiratory Drug Delivery (UK) Ltd. Dennis Sandell is a self-employed statistician and the owner of S5 Consulting; he is an independent consultant who was engaged by Philips on this project.

\section{References}

1. Lavorini F, and Fontana GA: Targeting drugs to the airways: the role of spacer devices. Expert Opin Drug Deliv. 2009;6:91-102.

2. Newman SP, Millar AB, Lennard-Jones TR, Morén F, and Clarke SW: Improvement of pressurised aerosol deposition with Nebuhaler spacer device. Thorax. 1984;39:935-941.

3. Corr D, Dolovich M, McCormack D, Ruffin R, Obminski $\mathrm{G}$, and Newhouse M: Design and characteristics of a portable breath-actuated particle size selective medical aerosol inhaler. J Aerosol Sci. 1982;13:1-7.

4. Rau JL: Practical problems with aerosol therapy in COPD. Respir Care. 2006;51:158-172.

5. Barry PW, Robertson CF, and O'Callaghan C: Optimum use of a spacer device. Arch Dis Child. 1993;69:693-694.

6. Newman SP, Woodman G, Morén F, and Clarke SW: Bronchodilator therapy with Nebuhaler: how important is the delay between firing the dose and inhaling? Br J Dis Chest. 1988;82:262-267.

7. Agnew JE: Physical properties and mechanisms of deposition of aerosols. In: SW Clarke, and D Pavia, (eds). Aerosols and the Lung: Clinical and Experimental Aspects. Butterworths, London; pp. 49-70, 1984.

8. Dolovich MA, MacIntyre NR, Anderson PJ, Camargo CA Jr, Chew N, Cole CH, Dhand R, Fink JB, Gross NJ, Hess DR, Hickey AJ, Kim CS, Martonen TB, Pierson DJ, Rubin BK, and Smaldone GC: Consensus statement: aerosols and delivery devices. American Association for Respiratory Care. Respir Care. 2000;45:589-596; Erratum in Respir Care. 2000;45:1416.

9. Barry PW, and O'Callaghan C: Poor output of salbutamol from a spacer device: effect of spacer static charge and multiple actuations. Thorax. 1994;49:402P.

10. O'Callaghan C, Lynch J, Cant M, and Robertson C: Improvement in sodium cromoglycate delivery from a spacer device by use of an antistatic lining, immediate inhalation, and avoiding multiple actuations of drug. Thorax. 1993;48: 603-606.
11. Berg E, Madsen J, and Bisgaard H: In vitro performance of three combinations of spacers and pressurized metered dose inhalers for treatment in children. Eur Respir J. 1998;12: 472-476.

12. Newman SP, Pavia D, Garland N, and Clarke SW: Effects of various inhalation modes on the deposition of radioactive pressurized aerosols. Eur J Respir Dis. 1982;63(Suppl 119):57-65.

13. Heslop K: How to use pressurised metered dose inhalers. Nurs Times. 2008;104(47):78-80.

14. Pool JB, Greenough A, Gleeson JGA, and Price JF: Inhaled bronchodilator treatment via the Nebuhaler in young asthmatic patients. Arch Dis Child. 1988;63:288-291.

15. Schultz A, Le Souëf T, Venter A, Zhang G, Devadason SG, and Le Souëf PN: Aerosol inhalation from spacers and valved holding chambers requires few tidal breaths for children. Pediatrics. 2010;126:e1493-e1498.

16. Hatley RHM, von Hollen D, Sandell D, and Slator L: In vitro characterization of the OptiChamber Diamond valved holding chamber. J Aerosol Med Pulm Drug Deliv. 2014; 27:S-24-S-36.

17. European Medicines Agency/Committee for Medicinal Products for Human Use (CHMP): Guideline on the requirements for clinical documentation for orally inhaled products (OIP) including the requirements for demonstration of therapeutic equivalence between two inhaled products for use in the treatment of asthma and chronic obstructive pulmonary disease (COPD) in adults and for use in the treatment of asthma in children and adolescents. European Medicines Agency, London, 22 January 2009. Doc. Ref. CPMP/EWP/4151/00 Rev. 1.

Received on May 21, 2013 in final form, November 11, 2013

Reviewed by: Elna Berg Sunalene Devadason

Address correspondence to: Ms. Lois Slator Respironics Respiratory Drug Delivery (UK) Ltd., a business of Philips Electronics UK Limited Chichester Business Park City Fields Way

Tangmere

Chichester

West Sussex

PO20 2FT, UK

E-mail: lois.slator@philips.com 